
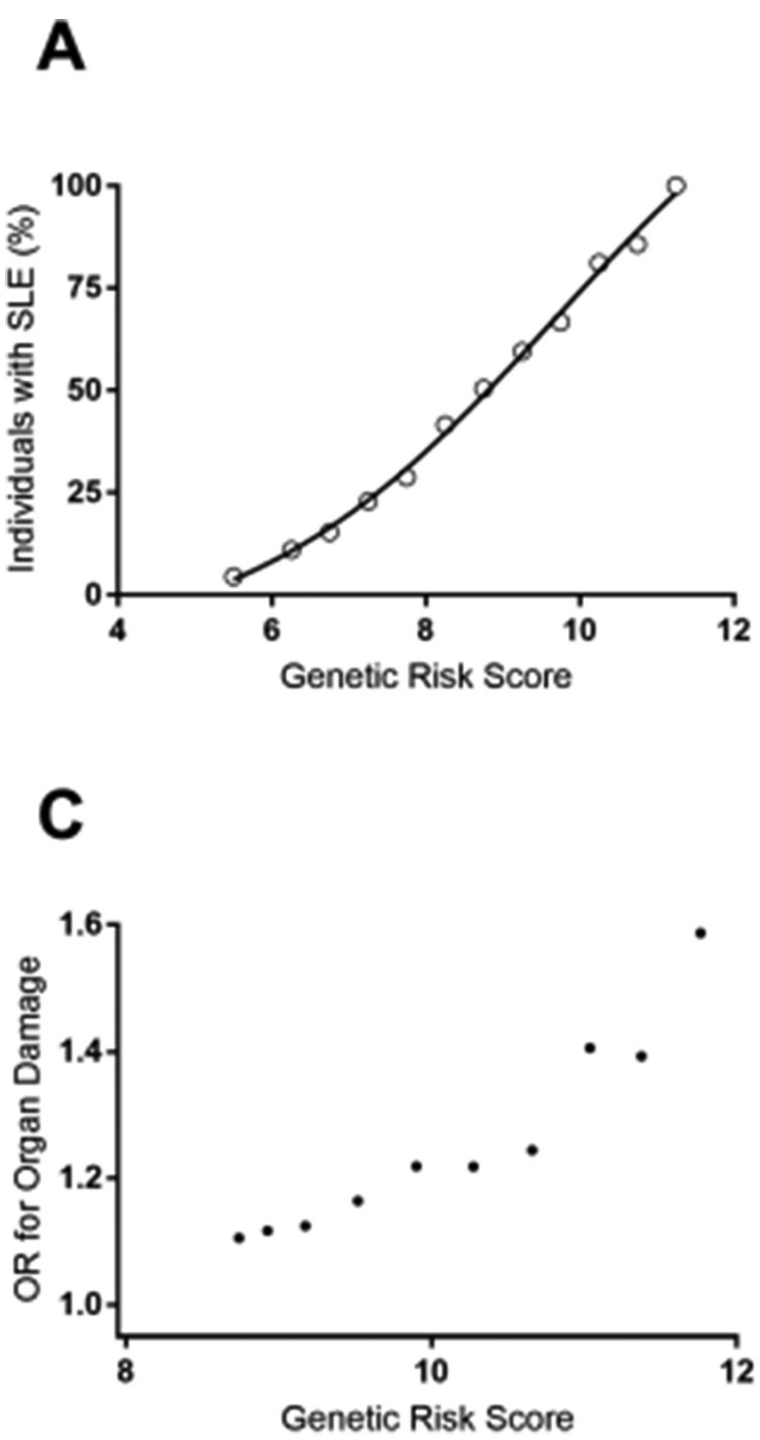

B

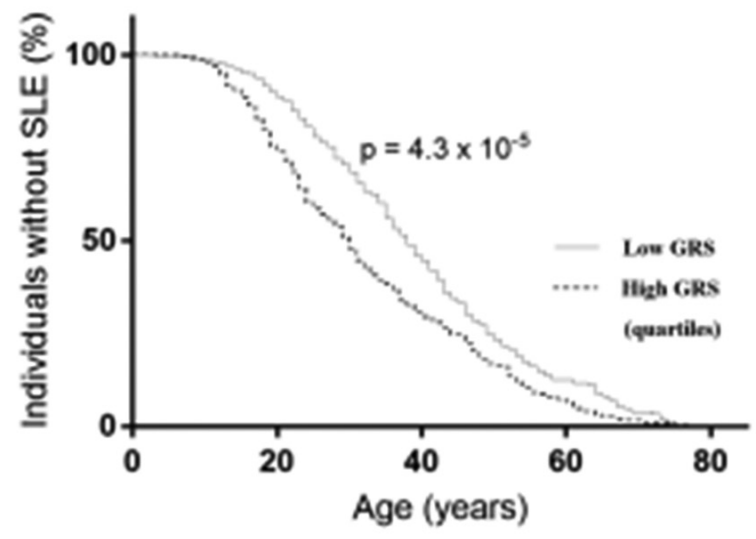

D

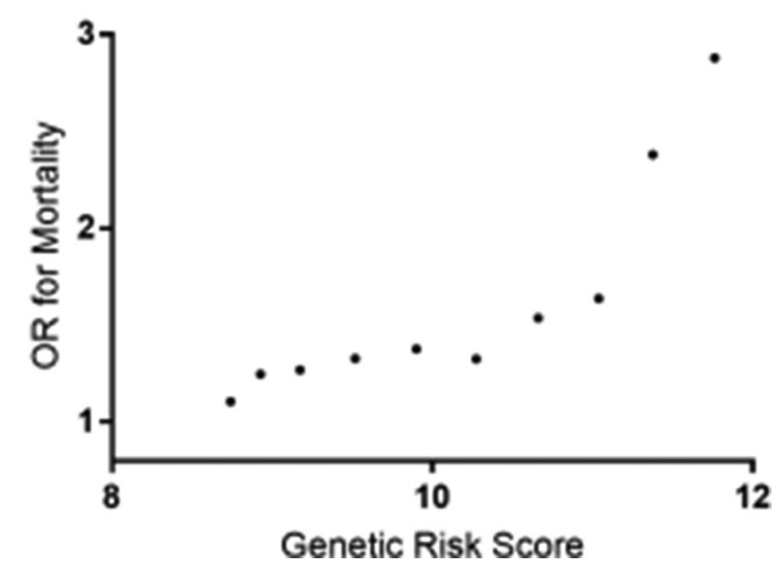

Abstract 207 Figure 1 Association of the genetic risk score with SLE onset, organ mortality. A) The prevalence of SLE in the patient-control population was plotted for groups with a GRS of $<6,6-6.5,6.5-7,7-7.5,7.5-8,8-8.5,8.5-9,9-9.5,9.5-10,10.5-11$ and $>11$. B) The survival until SLE onset was analysed for patients with a GRS in the extreme quartiles. $(n=500)$. C) IN regression analysis, the prevalence of organ damage in patients with a genetic risk score (GRS) above 7.0, 7.5, 8.0, 8.5, 9.0, 9.5, 10.0, 10.5, 11.0 and 11.5, respectively, was compared to a reference group with a GRS below 7.0. The odds ratio for each each group was plotted against the mean GRSs. Age was used as acovariate in each analysis D) Using the same method and groups as in C, odds ratio for mortality were plotted against mean GRSs.

survival. Our results indicate that genetic profiling may be useful for predicting outcomes in patients with SLE.

Funding Source(s): None

\section{8 (5R)-5-HYDROXYTRIPTOLIDE AMELIORATES NEPHRITIS IN LUPUS-PRONE MICE BY PREVENTING INFILTRATION OF IMMUNE CELLS}

${ }^{1}$ Jian-ping Zuo*, 'Shi-jun He, 'Ze-min Lin, ${ }^{2}$ Wei Tang. 'Laboratory of Immunopharmacology, State Key Laboratory of Drug Research, Shanghai Institute of Materia Medica, Chinese Academy of Sciences, Shanghai 201203, China; 'Laboratory of Immunopharmacology, Shanghai Institute of Materia Medica, Chinese Academy of Sciences, Shanghai 201203, China

10.1136/lupus-2019-|sm.208

Background (5R)-5-hydroxytriptolide (LLDT-8), a triptolide derivative with low toxicity, was previously reported to have strong immunosuppressive effects both in vitro and in vivo, but it remains unknown whether LLDT-8 has a therapy effect on systemic lupus erythematosus (SLE).

Methods In vivo, the therapeutic effects of LLDT-8 in MRL/ lpr mice were investigated. Ex vivo, the mechanisms of treatment were explored according to the immunologic correlates of disease. In vitro, human proximal tubule epithelial cell line and mouse mesangial cell line were used to evaluate the regulatory effects of LLDT-8 on chemokine expression.

Results Compared with the vehicle group, different clinical parameters were improved upon LLDT-8 treatment as follows: prolonged life span of mice, decreased proteinuria, downregulated blood urea nitrogen and serum creatinine, reduced glomerular IgG deposits, and ameliorated histopathology. A decreased expression of the inflammatory cytokines IFN- $\gamma$, IL17, IL- 6 , and TNF- $\alpha$ was also observed in the kidney of LLDT-8 treated MRL/lpr mice. Moreover, infiltration of $\mathrm{T}$ cells in the kidney was mitigated after LLDT-8 treatment, 
corresponding with decreased expression of related chemokines IP-10, Mig, and RANTES in the kidney. The proportion of macrophage and neutrophil cells and related chemokines expression was also reduced in kidneys of LLDT-8-treated mice. In the human proximal tubule epithelial cell line and mouse mesangial cell line, consistent with our in vivo experimental results, LLDT-8 suppressed the expression of related chemokines and IL-6.

Conclusions In summary, LLDT-8 has a therapeutic benefit for lupus nephritis via suppressing chemokine expression and inhibiting immune cell infiltration in kidneys of MRL/lpr mice.

Funding Source

This study was supported by National Science \& Technology Major Project 'Key New Drug Creation and Manufacturing Program,' China (2014ZX09101002-001).

\section{ATTAINMENT OF LOW DISEASE ACTIVITY AND REMISSION WITH ATACICEPT IN PATIENTS WITH SYSTEMIC LUPUS ERYTHEMATOSUS AND HIGH DISEASE ACTIVITY IN THE PHASE IIB ADDRESS II STUDY AND ITS LONG-TERM EXTENSION}

${ }^{1}$ Eric Morand, ${ }^{2}$ Joan T Merrill, ${ }^{3}$ David A Isenberg, ${ }^{4}$ Amy H Kao, ${ }^{4}$ Cristina Vazquez-Mateo, ${ }^{4}$ Stephen Wax, ${ }^{4}$ Peter Chang, ${ }^{4}$ Kishore Pudota, ${ }^{5}$ Cynthia Aranow, ${ }^{6}$ Daniel J Wallace. ${ }^{1}$ Monash University, Melbourne, Australia; ${ }^{2}$ Oklahoma Medical Research Foundation; ${ }^{3}$ University College London, London, UK; ${ }^{4}$ EMD Serono Research \& Development Institute, Inc. (a business of Merck KGaA, Darmstadt, Germany), Billerica, MA, USA; ${ }^{5}$ Feinstein Institute for Medical Research; ${ }^{6}$ Cedars-Sinai Medical Center, David Geffen School of Medicine, UCLA, Los Angeles, CA, USA

10.1136/lupus-2019-Ism.209
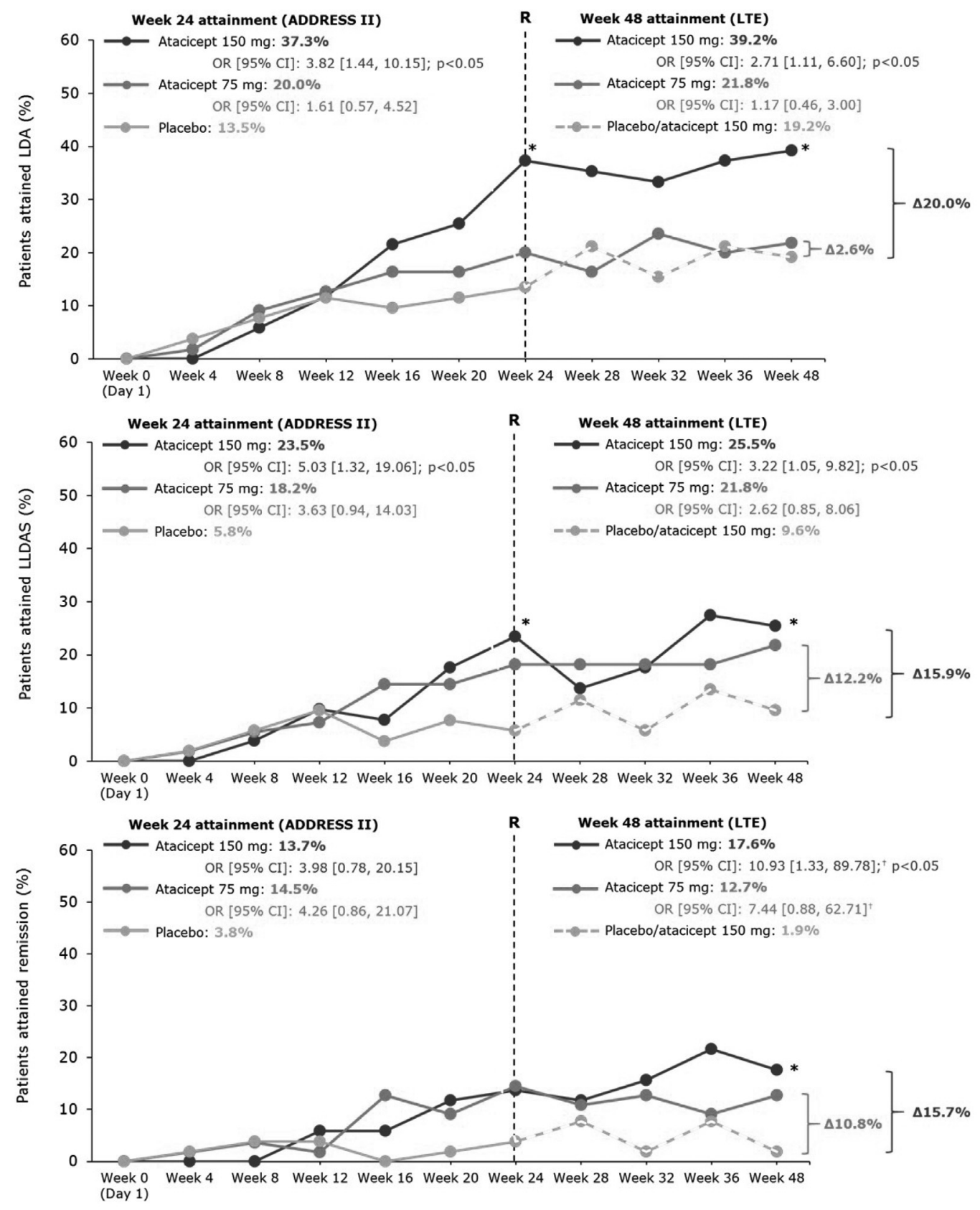

${ }^{*} \mathrm{p}<0.05$ versus placebo ( 24 weeks) or placebo/atacicept $150 \mathrm{mg}$ ( 48 weeks); †Results should be interpreted with caution due to the wide CI; CI, Confidence interval; LDA, Low disease activity; LLDAS, Lupus Low Disease Activity State; LTE, Long-term extension; OR, Odds ratio

\section{Abstract 209 Figure 1}

